\title{
Post-traumatic-stress-disorder PTSD
}

\section{Opinion}

Dentists and especially Pediatric Dentists face the clinical manifestations of the PTSD on a daily basis. The cortex in the Central Nervous System is where this disorder is generated and interpreted, the temporal cortex of the brain after receiving information from sub-cortical sites within the brain. These sites were gathering input informations from outside environment through sensory organs. Exposure to previous traumatic experience will trigger the same old, negative and painful memory whenever a similar situation is perceived or one of its stimuli components. Some attempts, like eliminating the dental office smell, changing the classic white lab coats color and design to more attractive ones or adding some cartoon figures, were clearly successful reducing the PTSD manifestations in clinical practice. However, other means were quite successful, like the usage of audio and visual effects. Some techniques utilized headphones and devices to show videos, play music, and recently the usage of the VR devices. These distractions supposedly hide the semantic memory, minimize the effect of external stimulus input, and mask or decrease the output of the old established pictures. Recent studies showed that when a patient is a witness or a victim of a painful or tragic circumstances, he/she will react in a negative way as soon as he/she enter the dental office, this apply especially on children. The studies also suggest how we can possibly prevent this syndrome to appear (the unpleasant description of a dental scenes transmitted from parents to their children remain a challenge). The medical approach to face this problem stands on two methods, the first is to suppress the reactions by giving oral Medazolam and in some studies Barbiturates and/or Antihistamine composition, assisted intra-venous sedation (conscious sedation) or by applying the G.A. The second consists of
Volume 7 Issue 2 - 2017

\author{
Sadek Bakdach \\ Department of Pediatric Dentistry, Paris Academy, USA
}

Correspondence: Sadek Bakdach, Department of Pediatric Dentistry, Paris Academy, 4058 State St, Saginaw, MI 48603, USA, Email sadekbakdach@gmail.com

Received: April 12, 2017| Published: April 25, 2017

trying to build a new memory and to replace the old one and this is a very long procedure and requires many gradually progressing sessions to a neutral one without any success assurance and of course the overall clinical conditions will guide our choice. The PTSD is composed of two components the brain and the body, so let's try to eliminate the body component by administrating Betta-blocker which act on the synapsis in the Central Nervous System. The question is can we administer Betta-blocker to children? How and under what conditions? Is it really effective?

\section{Acknowledgments}

None.

\section{Conflicts of interest}

The author declares there are no conflicts of interest. 